\title{
Long non-coding RNAs as prognostic biomarkers in papillary renal cell carcinoma
}

\author{
FEILONG YANG, YIMENG SONG, LIYUAN GE, GUOJIANG ZHAO, CHENG LIU and LULIN MA \\ Department of Urology, Peking University Third Hospital, Beijing 100191, P.R. China
}

Received January 3, 2019; Accepted June 13, 2019

DOI: $10.3892 / \mathrm{ol} .2019 .10684$

\begin{abstract}
The aim of the present study was to identify long non-coding RNA (lncRNA)-based prognostic biomarkers in papillary renal cell carcinoma (pRCC). lncRNA expression data and corresponding clinical data from patients with $\mathrm{pRCC}$ were obtained from The Cancer Genome Atlas. R software and packages were used for data analysis. Univariate Cox regression analysis and least absolute shrinkage and selection operator regression were performed to identify key lncRNAs, which were then used to construct a prognostic model using multivariate Cox regression analysis. Patients were divided into high- and low-risk groups, and Kaplan-Meier (KM) survival curves and time-dependent receiver operating characteristic (ROC) curves were plotted. The $\mathrm{C}$-index was calculated to estimate the model's prognostic power. The hazard ratio (HR), 95\% confidence interval (CI), and statistical significance of each key IncRNA were also calculated by multivariate Cox regression. Based on the result of the multivariate Cox regression analysis, KM survival plots were plotted for each significantly associated lncRNA. The subcellular locations of the prognostic biomarkers were predicted using lncRNAMap and lncLocator. A total of 17 lncRNA signatures were identified as key lncRNAs. Overall survival rate was significantly higher in the low-risk group compared with the high-risk group. The areas under the ROC curve were 0.93 (3-year ROC) and 0.902 (5-year ROC), and the C-index was 0.915. A forest plot was used to illustrate the HR and 95\% CI of key lncRNAs. KM survival analysis revealed the prognostic significance of two protective biomarkers, AC024022.1 and GAS6-AS1, and three adverse biomarkers, AC087379.2, AL352984.1, and AL499627.1. It was predicted that AC024022.1 and AC087379.2 may be located in the cytoplasm and GAS6-AS1 may be located in the cytosol. The present study may contribute to the management of pRCC
\end{abstract}

Correspondence to: Dr Lulin Ma or Dr Cheng Liu, Department of Urology, Peking University Third Hospital, 49 North Garden Road, Haidian, Beijing 100191, P.R. China

E-mail:malulin@medmail.com.cn

E-mail: chengliu@bjmu.edu.cn

Key words: long non-coding RNAs, prognostic biomarker, papillary renal cell carcinoma, bioinformatics and serve as a foundation for further investigations into the underlying mechanism of tumorigenesis and progression of pRCC.

\section{Introduction}

According to Globocan 2018, 403,262 new cases of renal cell carcinoma ( $\mathrm{RCC}$ ) were diagnosed in 185 countries and these accounted for $2.2 \%$ of 36 types of cancer (1). The number of deaths associated with RCC was 175,098 , accounting for $1.8 \%$ of deaths associated with 36 types of cancer (1). Papillary RCC (pRCC) accounts for 10-20\% of all RCC cases (2). At present, surgery is the preferred therapeutic option for localized and locally advanced pRCC, and several agents, including anti-vascular endothelial growth factor drugs and mTOR inhibitors may be considered when treating advanced and metastatic pRCC (2). At present, pathological stage based primarily on clinicopathological data is still the most important factor influencing the prognosis of renal carcinoma, but it does not reflect the biological heterogeneity of the cancer (3). In recent years, with the development of molecular biological techniques and bioinformatics, new biomarkers have the potential of becoming effective and specific prognostic factors for different types of cancer, including pRCC.

Long non-coding RNAs (lncRNAs) are RNA transcripts that do not encode proteins and are $>200$ nucleotides (nt) in length. IncRNAs are a diverse class of RNAs, distinct from mRNAs, and are involved in a number of important biological phenomena, including imprinting genomic loci, shaping chromosome conformation and allosterically regulating enzymatic activity (4-6). IncRNAs account for the largest portion of the mammalian non-coding transcriptome (7). Specific lncRNA expression patterns, such as overexpression, depletion, or mutations of lncRNA genes are associated with numerous human diseases $(7,8)$. Altered expression of specific lncRNAs may promote tumor formation, progression and metastasis in many malignancies, including RCC (8-10). Based on the features of cancer, lncRNAs have been suggested to serve important roles in their tumorigenesis, progression, treatment, and prognosis. However, the functions of most of the lncRNAs are not well understood.

In the present study, lncRNA-based prognostic biomarkers were identified and a prognostic model of pRCC was constructed. The IncRNA expression profiles between pRCC tissues and corresponding normal kidney tissues from The 
Cancer Genome Atlas (TCGA) were compared. Key lncRNAs were screened by univariate Cox regression and least absolute shrinkage and selection operator (LASSO) regression, followed by multivariate Cox regression analysis to establish the prognostic model using the obtained lncRNA expression data and corresponding clinical data of patients with pRCC. The Kaplan-Meier (KM) survival curves, ROC curves and the $\mathrm{C}$-index were determined to estimate the prognostic power of the model. In addition, the hazard ratio (HR) and corresponding P-value of each key lncRNA were calculated by multivariate Cox regression. Based on results of the multivariate Cox regression analysis, KM survival analysis for each significant key lncRNA was performed.

The results of the present study may contribute to the management of pRCC and improve our understanding of the underlying molecular mechanisms in tumorigenesis and progression of pRCC.

\section{Materials and methods}

lncRNA expression data and clinical data of patients with $p R C C$. IncRNA expression data and corresponding clinical data of patients with pRCC were obtained from the TCGA database (https://portal.gdc.cancer.gov/) (11). The version of the dataset was: Data Release 14.0-December 18, 2018. A total of 32 cancer-adjacent normal tissues and 289 cancer tissues from patients with pRCC were included. R software (version 3.4.4) (12), RStudio 1.2.1335-Windows 7+ (64-bit) (13) and $\mathrm{R}$ packages were used to prepare and analyze the corresponding data.

Differential expression analysis. The R package 'edgeR' (14) was utilized to screen differentially expressed lncRNAs in pRCC tissues compared with normal kidney samples. A false discovery rate (FDR) $<0.05$ and $\mid \log _{2} \mathrm{FCl}>2$ were used as the cutoff criteria for the differential expression, and values beyond these cutoffs were considered statistically significant. The differentially expressed lncRNAs were plotted using a volcano plot and heatmap using the R packages 'ggplot2' (cran.r-project. org/web/packages/ggplot2/index.html) and 'pheatmap' (cran.r-project.org/web/packages/pheatmap/index.html).

Univariate Cox regression. To determine the association between the expression of differentially expressed lncRNAs and overall survival (OS) rate, univariate Cox regression was performed using the 'survival' package (cran.r-project. org/web/packages/survival/index.html). $\mathrm{P}<0.05$ was considered to indicate a statistically significant difference. Survival data, including the survival time and survival status were merged with the expression data of the differentially expressed IncRNAs. Univariate Cox regression was then performed and statistically significant lncRNAs were identified and used for LASSO regression.

LASSO regression. To identify key lncRNAs with better performance parameters and to avoid overfitting, only statistically significant lncRNAs based on the results of the univariate Cox regression analysis were used for further LASSO regression using the 'glmnet' (https://cran.r-project. org/web/packages/glmnet/index.html) and 'survival' packages.
Similarly, the survival and expression data of the statistically significant lncRNAs from the results of the univariate Cox regression analysis were merged. Using the $\mathrm{R}$ packages 'glmnet' and 'survival', and the LASSO algorithm, the lncRNAs involved in the prognosis of patients with pRCC were selected by shrinkage of the regression coefficient, through imposing a penalty proportional to their size (15). As a result, most potential indicators were shrunk to zero and a relatively small number of indicators with a weight $\neq 0$ were left (17 lnRNAs).

Multivariate Cox regression. To further establish a prognostic model and to determine the relationship between the key lncRNAs based on the LASSO regression analysis results and the OS time, multivariate Cox regression analysis was performed using the 'survival' package, which calculated the risk score for each patient. All the patients were divided into high- and low-risk groups based on the median cutoff point of the risk scores (median, 0.76). In addition, the HR, 95\% CI and statistical significance of each key lncRNA was calculated and illustrated using a forest plot via the R package 'survminer' (cran.r-project. org/web/packages/survminer/index.html). $\mathrm{P}<0.05$ was considered to indicate a statistically significant difference. A heatmap was used to illustrate the expression levels of the key lncRNAs in both the groups.

ROC curve and $C$-index. To evaluate the prognostic power of the model, the 3-year and 5-year ROC curves were generated and the area under the curves (AUCs) were calculated using the 'survival' and 'timeROC' (cran.r-project.org/web/packages/timeROC/index.html) packages. An AUC of $<0.5$ was considered insignificant, while an AUC of 0.51-0.7, 0.71-0.9, and $>0.9$ were considered low, moderate, and high accuracy for the prognostic model, respectively. Furthermore, the $\mathrm{C}$-index was calculated using the 'survival' package. The evaluation criterion of the C-index was similar to that of the AUC.

KM survival analysis. The KM survival curve was performed for the high- and low-risk groups using the 'survival' package. KM survival curves were plotted individually for each key lncRNA obtained in the multivariate Cox regression analysis.

Prediction of subcellular location. As the function of lncRNA is associated with its subcellular localization, the sequences of the prognostic biomarkers were obtained from the lncRNAMap website (16). The obtained sequences were entered into the IncLocator website to predict the subcellular localization of the IncRNA (17). The results were obtained as a score for each potential subcellular location for each lncRNA, including the cytoplasm, nucleus, ribosome, cytosol and exosome. Finally, a location was predicted for each lncRNA, using a function of the website.

\section{Results}

Identification of differentially expressed lncRNAs in pRCC. A total of 14,447 lncRNAs were extracted from the database, and 8,044 lncRNAs were identified as expressed genes. Moreover, 1,001 lncRNAs were differentially expressed in pRCC tissues compared with normal tissues according to the screening criterion (FDR $<0.05$ and $\log _{2} \mathrm{FCl}>2$ ). Of these, 546 
were upregulated $\left(\log _{2} \mathrm{FC}>2\right)$ and 455 were downregulated $\left(\log _{2} \mathrm{FC}<-2\right.$; Fig. 1A).

Establishment and evaluation of the prognostic model. Univariate Cox regression analysis was first performed for the 1,001 differentially expressed lncRNAs. Of these, the differential expression of 348 was considered statistically significant. LASSO regression was carried out on these 348 lncRNAs, and 17 key lncRNAs were identified (Fig. 1B and C). Multivariate Cox regression was performed to establish the prognostic model based on the 17 key lncRNAs. The risk scores for all patients were calculated, and patients were divided into high- and low-risk groups based on 0.76, the median cutoff point of the scores. To evaluate the prognostic model, the 3 -year and 5-year ROC curves were plotted and the C-index was calculated. The AUC values were 0.93 (3-year ROC) and 0.902 (5-year ROC), and the C-index was 0.915 (Fig. 2A). Furthermore, KM survival analysis revealed that the OS rate was significantly worse in the high-risk group compared with the low-risk group ( $\mathrm{P}<0.001$; Fig. 2B). The HR, 95\% CI, and its statistical significance for each of the 17 key lncRNAs were determined using multivariate Cox regression, and the results are presented as a forest plot in Fig. 3 .

KM survival analysis for key lncRNAs. Based on the results of the multivariate Cox regression, six lncRNAs, AC024022.1, AC087379.2, AL352984.1, AL499627.1, GAS6-AS1 and MEG3, were used for further KM survival analysis. AC024022.1 and GAS6-AS1 were identified as protective prognostic biomarkers, while AC087379.2, AL352984.1 and AL499627.1 were identified as adverse prognostic biomarkers (Fig. 4A-E). MEG3 may additionally be a potential adverse prognostic biomarker, although the difference was not statistically significant (Fig. 4F).

Subcellular location prediction. IncLocator predicted that AC024022.1 and AC087379.2 were located in the cytoplasm and GAS6-AS1 was located in the cytosol (Table I). The subcellular localizations of AL352984.1 and AL499627.1 were not predicted as IncRNAMap website did not possess a record of their sequences. The sequence information of AC024022.1, AC087379.2 and GAS6-AS1 obtained from IncRNAMap are presented in Table S1.

\section{Discussion}

Transcription is classified into protein-coding and non-coding transcripts. Coding genes are estimated to account for $<2 \%$ of the human transcripts, while non-coding RNA transcripts account for $\sim 98 \%$ of transcriptome $(18,19)$. Among the non-coding transcripts, IncRNAs are a loosely classified group of long RNA transcripts ( $>200$ nucleotides in length) with no apparent protein-coding potential (4). However, lncRNAs not only have similar structures as protein-coding genes such as introns and exons, but also possess a wide range of biological functions involved in a variety of cellular activities (19). For instance, lncRNAs are involved in cell proliferation, survival, apoptosis and movement by regulating gene expression at the epigenetic, transcriptional, and post-transcriptional levels $(20,21)$. The functions of IncRNA
Table I. Prediction of subcellular locations of long non-coding RNAs.

\begin{tabular}{lccc}
\hline Location & AC024022.1 & AC087379.2 & GAS6-AS1 \\
\hline Cytoplasm & 0.512660116 & 0.603843419 & 0.139349109 \\
Nucleus & 0.363217766 & 0.097721928 & 0.107388576 \\
Ribosome & 0.016871894 & 0.073857973 & 0.094522592 \\
Cytosol & 0.06045974 & 0.073769925 & 0.640534382 \\
Exosome & 0.046790483 & 0.150806755 & 0.01820534 \\
Predicted & Cytoplasm & Cytoplasm & Cytosol \\
location & & & \\
\hline
\end{tabular}

The numbers listed in the table represent the scores for the corresponding subcellular locations. The number of the score ranges from 0 to 1 .

are primarily executed by serving as a: i) Molecular signal that can respond to intracellular or extracellular signals and act as a regulator for specific signal pathways; ii) molecular decoy that may combine certain RNAs or proteins that may be removed from a particular subcellular location, thus abrogating or attenuating their normal function; iii) molecular guides for proteins to a specific mRNA or chromosome locus, influencing genetic transcription, mRNA stability and translation; and iv) molecular scaffold, which can bind multiple molecules for specific functions (20). The subcellular localization of lncRNA can be used to predict its functions (20). For example, if a lncRNA is located in the nucleus, it may be involved in chromatin regulation, gene transcription and alternative splicing of transcripts, whereas if it is in the cytoplasm, it may serve as a competing endogenous (ce)RNA or regulate mRNA stability or translation.

At present, there is increasing evidence that aberrations within IncRNAs such as overexpression, deficiency or mutations drive important cancer phenotypes (18), including tumor formation, progression and metastasis in many types of cancer $(7,8,10,22,23)$. In addition, certain aberrant lncRNAs are additionally associated with increasing the malignant biological behaviors of cancer cells, clinicopathological features and poor prognosis of cancers (23-25). In clear cell RCC, some aberrant lncRNAs, such as PVT1, TUG1, NEAT1, and PANDAR, can serve as prognostic indicators (26-29). However, to the best of our knowledge, there are no previous studies investigating the association between IncRNA and the prognosis of pRCC.

In the present study, to identify potentially valuable lncRNAs for improving prognosis of patients with pRCC, existing lncRNA expression data along with corresponding clinical data in TCGA was analyzed to identify the key IncRNAs using univariate Cox regression and LASSO regression analyses. A prognostic model was then constructed by multivariate Cox regression based on the identified key lncRNAs. All the patients were divided into high- and low-risk groups and the KM survival curves, ROC curves and C-index calculation were performed, and the prognostic power of the model was estimated. In addition, the HR and $95 \% \mathrm{CI}$ of each key IncRNA were also calculated by multivariate Cox regression analysis. Based on these results, KM survival analysis was performed for each significant key lncRNA. A total of 
A

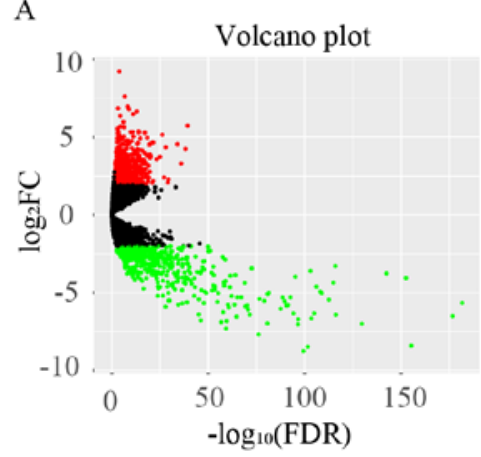

B

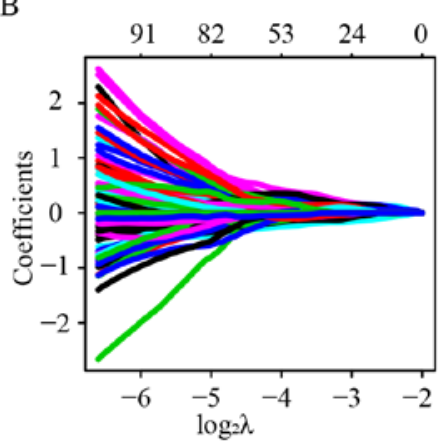

C

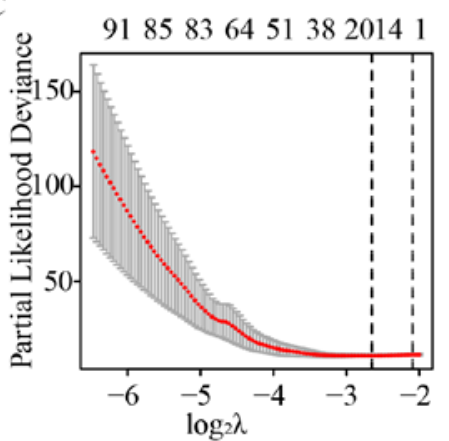

Figure 1. Identification of 17 key lncRNA signatures using univariate Cox and LASSO regression. (A) Volcano plot of all lncRNAs. A total of 1,001 differ-

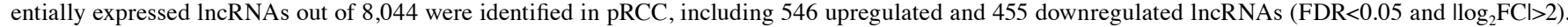
(B) Tuning parameter and (C) variable selection using the LASSO model. The numbers on the top of the figures indicated the number of the candidate lncRNAs for the corresponding lambda $(\lambda)$ value in LASSO regression. A total of 348/1,001 significant lncRNAs from the result of the univariate Cox regression were used for the LASSO regression, and 17 key lncRNAs were identified by multivariate Cox regression. pRCC, papillary renal cell carcinoma; lncRNAs, long non-coding RNAs; LASSO, least absolute shrinkage and selection operator; FC, fold change; FDR, false discovery rate.

A

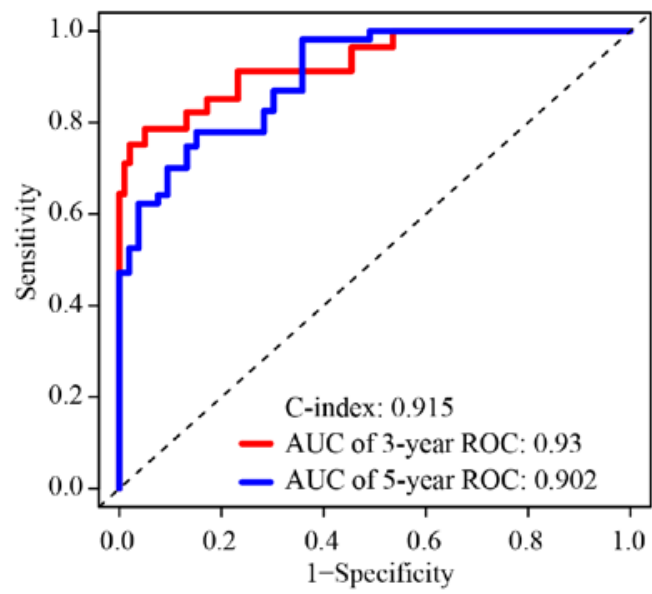

B

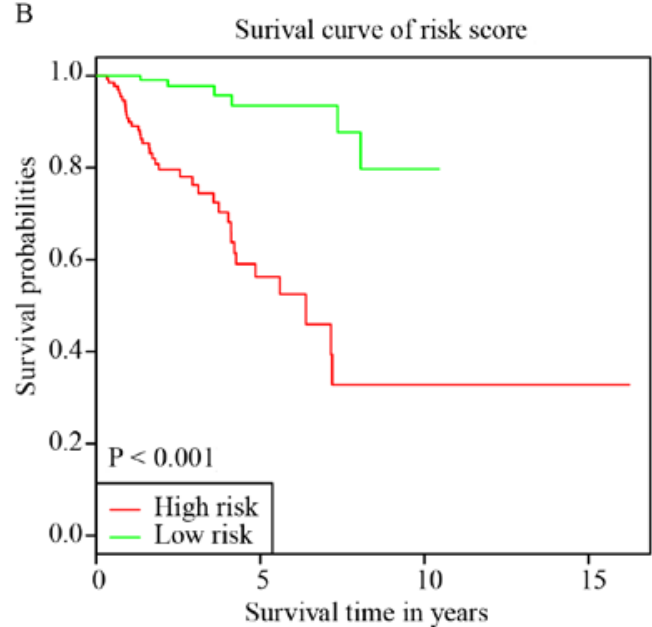

$\mathrm{C}$

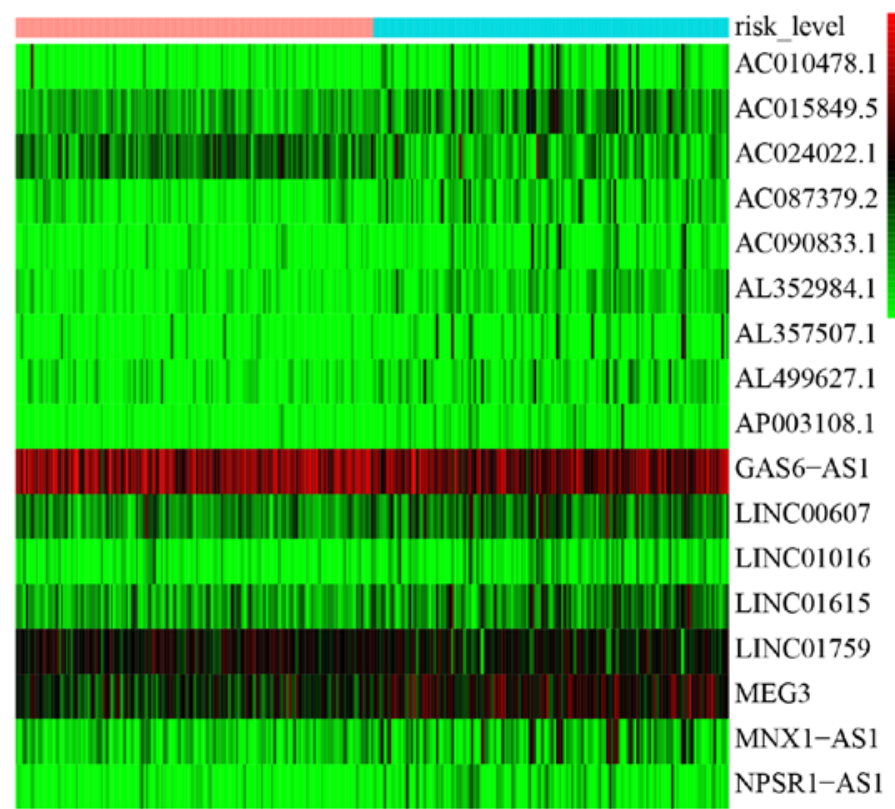

Figure 2. Establishment and evaluation of the prognostic model. (A) 3-year and 5-year ROC curves and C-index of the 17 key lncRNAs. AUC=0.93 (3-year ROC) and AUC=0.902 (5-year ROC), C-index=0.915. (B) KM survival curve of the risk level. Overall survival rate of patients with low-risk scores was significantly higher $(\mathrm{P}<0.001)$. (C) Heatmap of the expression levels of the 17 key lncRNAs in the high-risk group and low-risk group. Red and green represent increased and decreased normalized expression value of the lncRNAs in all patients, respectively. KM, Kaplan-Meier; ROC, receiver operating characteristic; AUC, area under curve; lncRNAs, long non-coding RNAs. 


\section{Hazard ratio}

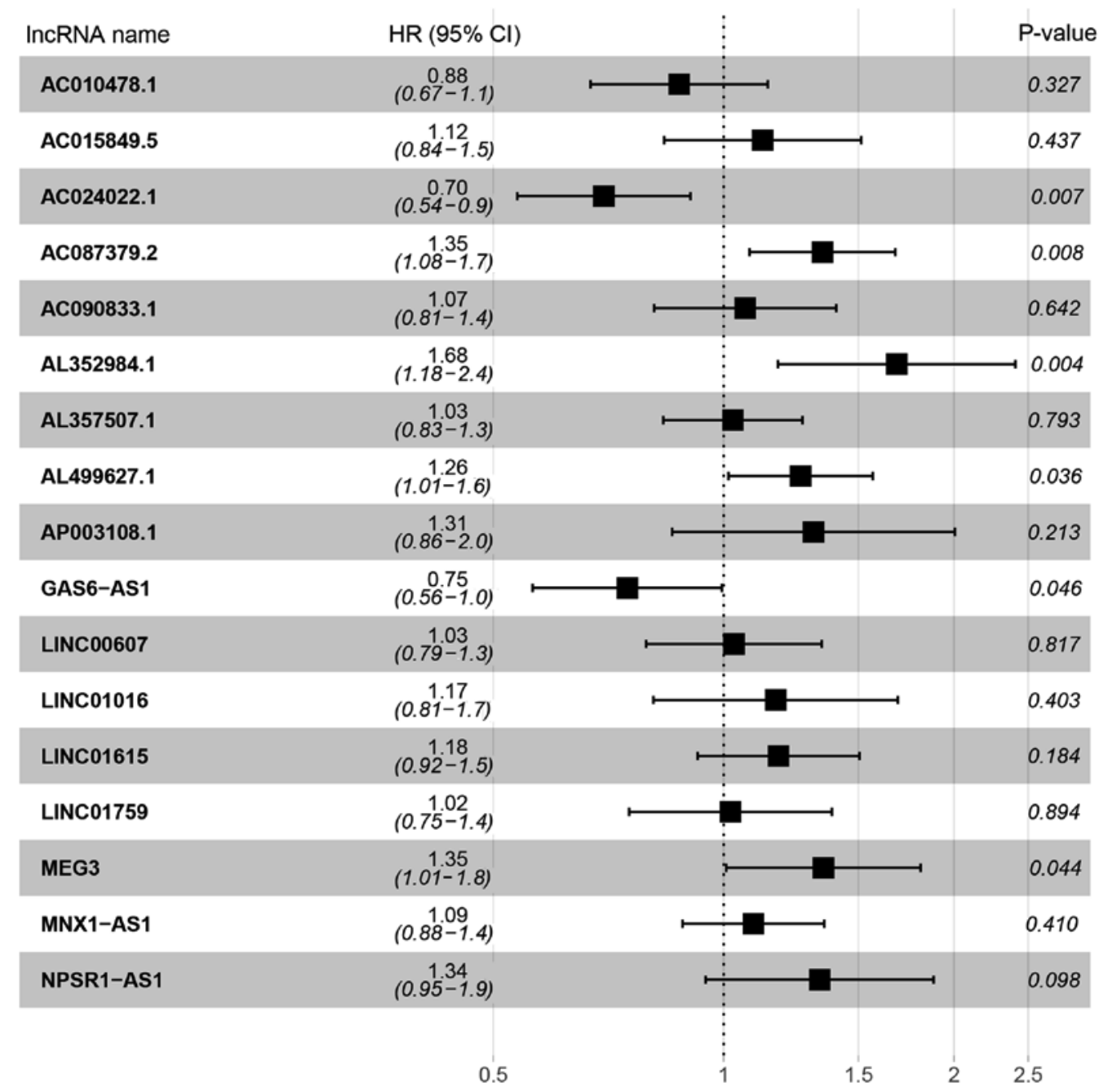

Figure 3. HR and 95\% CI of the 17 key lncRNAs by the multivariate Cox regression. A total of 285 patients was included in the analysis. Of the 17 key lncRNAs, six had a significant influence on the OS rate of patients with pRCC, including AC024022.1, AC087379.2, AL352984.1, AL499627.1, GAS6-AS1 and MEG3. HR, hazard ratio; CI, confidence interval; lncRNAs, long non-coding RNAs; OS, overall survival; pRCC, papillary renal cell carcinoma.

1,001 differentially expressed lncRNAs were identified, of which 546 were upregulated and 455 were downregulated. Furthermore, univariate Cox regression and LASSO regression analyses identified 17 key lncRNAs, which were used to establish the prognostic model. Furthermore, the results of the KM survival analysis revealed that patients in the high-risk group had significantly lower OS rate compared with the low risk group. Based on the multivariate Cox regression analysis, six lncRNAs were identified as potential prognostic biomarkers. The prognostic values of five lncRNAs, AC024022.1, AC087379.2, AL352984.1, AL499627.1, and GAS6-AS1 (all $\mathrm{P}<0.05$ ), were validated by KM survival analysis.

Subsequently, the nucleotide database was retrieved from the lncRNA map. The lncRNA AC024022.1 (ENSG00000250781) is the sequence of 'Homo sapiens BAC clone RP11-63A11'. The lncRNA AC087379.2 (ENSG00000254695) is defined as 'Homo sapiens chromosome 11, clone RP11-396020'. The lncRNA AL352984.1 (ENSG00000258386) is the sequence of Homo sapiens BAC R-187E13 of library RPCI-11 from chromosome 14. The IncRNA AL499627.1 (ENSG00000260542) is the sequence from clone RP13-379024 on human chromosome 20. GAS6AS1 (ENSG00000233695) is defined as Homo sapiens GAS6 antisense RNA-1. Decreased expression of 1ncRNA GAS6-AS1 predicted a poor prognosis in patients with non-small cell lung cancer (NSCLC) (30), consistent with the present study. Decreased expression of GAS6-AS1 predicted a worse OS rate, suggesting that GAS6-AS1 is a protective biomarker for the prognosis of pRCC. Correct subcellular localization of lncRNA transcripts is important for their functions. Therefore, the subcellular locations of the lncRNA transcripts were predicted using lncLocator (17) based on the sequences acquired from IncRNAMap (16). It was predicted that AC024022.1 (ENSG00000250781) and AC087379.2 (ENSG00000254695) were likely located in the cytoplasm, and GAS6-AS1 (ENSG00000233695) were likely located in the cytosol. The subcellular localization of AL352984.1 (ENSG00000258386) and AL499627.1 (ENSG00000260542) were not predicted because their sequence information was not present on lncRNAMap. As previously mentioned, if a lncRNA was located in the cytoplasm or cytosol, it may serve as a ceRNA, and regulate mRNA stability or translation (20). 

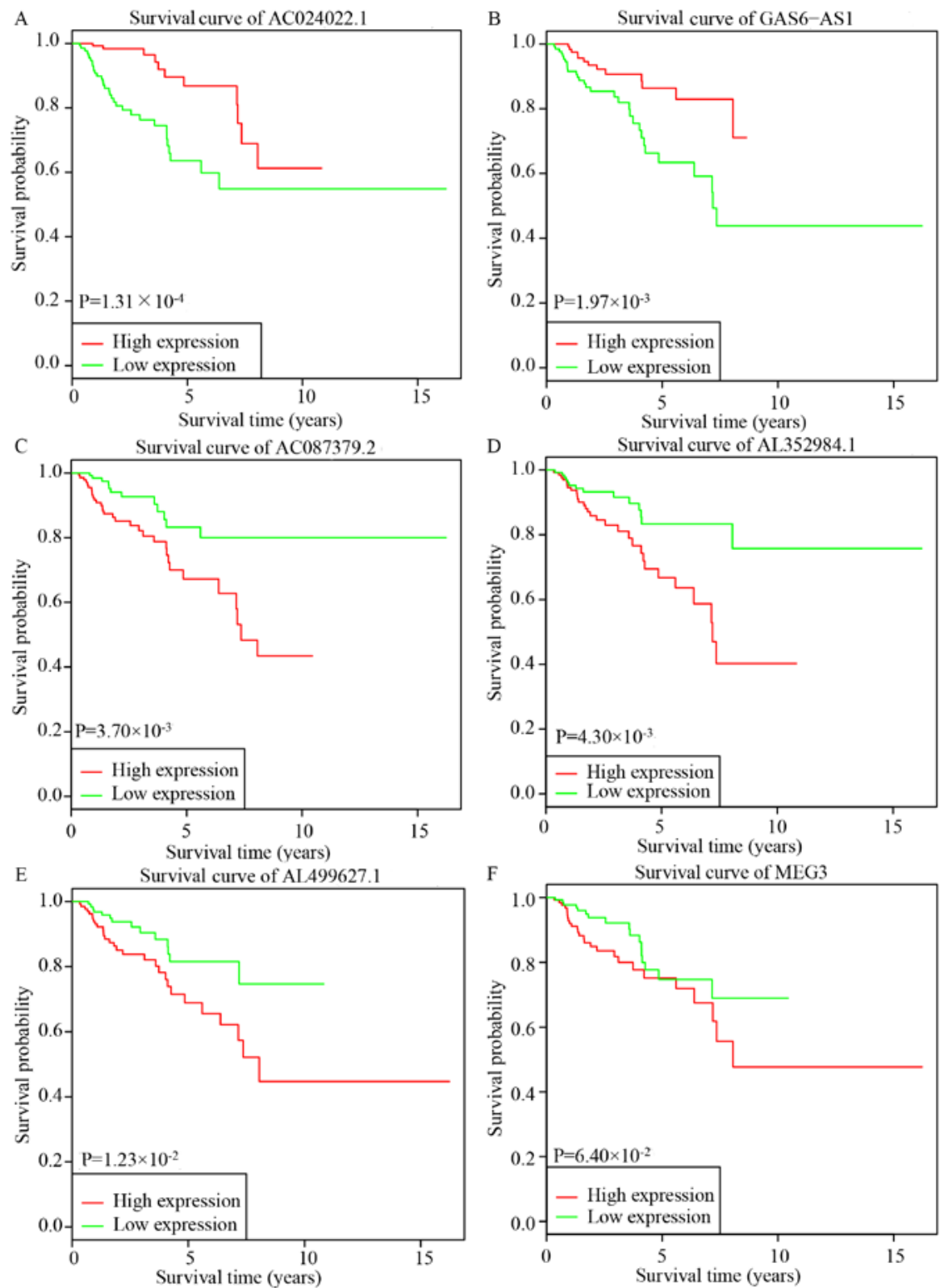

Figure 4. KM survival curves of the six significant key lncRNAs identified by multivariate Cox regression. (A) AC024022.1 and (B) GAS6-AS1 were identified as protective prognostic biomarkers, while (C) AC087379.2, (D) AL352984.1 and (E) AL499627.1 were identified as adverse prognostic biomarkers (all $\mathrm{P}<0.05)$. (F) MEG3 did not adversely affect OS rate $(\mathrm{P}=0.06)$. KM, Kaplan-Meier; pRCC, papillary renal cell carcinoma; lncRNAs, long non-coding RNAs; OS, overall survival.

Therefore, we hypothesized that lncRNAs AC024022.1 (ENSG00000250781), AC087379.2 (ENSG00000254695), and GAS6-AS1 (ENSG00000233695) acted as ceRNA, and served a role in regulating the stability or translation of mRNA. Unfortunately, to the best of our knowledge, there were no previous studies for these IncRNAs, and further studies are required to investigate the features, functions, and mechanisms of them.

In summary, a 17-IncRNA signature was constructed by mining the TCGA database, and identified two protective lncRNAs, AC024022.1 (ENSG00000250781) and GAS6-AS1 (ENSG00000233695), and three potentially tumorigenic lncRNAs, AC087379.2 (ENSG00000254695), AL352984.1 (ENSG00000258386) and AL499627.1 (ENSG00000260542). AC024022.1 (ENSG00000250781) and AC087379.2 (ENSG00000254695) might be located in the cytoplasm while GAS6-AS1 (ENSG00000233695) might be located in the cytosol. The present study identified novel lncRNAs, which may serve as biomarkers for the prognosis of pRCC and these findings may contribute to the management or further investigation into the underlying mechanisms of pRCC.

\section{Acknowledgements}

Not applicable.

\section{Funding}

The present study was funded by The National Natural Science Foundation of China (grant nos. 81711530048 and 81572515), Beijing Municipal Science \& Technology Commission (grant no. Z151100003915105). 


\section{Availability of data and materials}

The datasets generated and/or analyzed during the current study are available in the TCGA repository (https://portal. gdc.cancer.gov/). The version of the dataset was: Data Release 14.0-December 18, 2018.

\section{Authors' contributions}

LM and CL contributed to the design of the study, acquisition of funding, and general supervision. FY contributed to data analysis and wrote the manuscript. LG and GZ contributed to acquisition and interpretation of data, and revised the manuscript critically for important intellectual content. YS offered guidance, interpreted the data for the work, and reviewed the article for publication. All authors have read and approved the final manuscript.

\section{Ethics approval and consent to participate}

Not applicable.

\section{Patient consent for publication}

Not applicable.

\section{Competing interests}

The authors declare that they have no competing interests.

\section{References}

1. Bray F, Ferlay J, Soerjomataram I, Siegel RL, Torre LA and Jemal A: Global cancer statistics 2018: GLOBOCAN estimates of incidence and mortality worldwide for 36 cancers in 185 countries. CA Cancer J Clin 68: 394-424, 2018.

2. Courthod G, Tucci M, Di Maio M and Scagliotti GV: Papillary renal cell carcinoma: A review of the current therapeutic landscape. Crit Rev Oncol Hematol 96: 100-112, 2015.

3. Cheng P: A prognostic 3-long noncoding RNA signature for patients with gastric cancer. J Cell Biochem 119: 9261-9269, 2018.

4. Quinn JJ and Chang HY: Unique features of long non-coding RNA biogenesis and function. Nat Rev Genet 17: 47-62, 2016.

5. Rinn JL and Chang HY: Genome regulation by long noncoding RNAs. Annu Rev Biochem 81: 145-166, 2012.

6. Ponting CP, Oliver PL and Reik W: Evolution and functions of long noncoding RNAs. Cell 136: 629-641, 2009.

7. Esteller M: Non-coding RNAs in human disease. Nat Rev Genet 12: 861-874, 2011.

8. Martens-Uzunova ES, Bottcher R, Croce CM, Jenster G, Visakorpi $T$ and Calin GA: Long noncoding RNA in prostate, bladder, and kidney cancer. Eur Urol 65: 1140-1151, 2014.

9. Jin Y, Feng SJ, Qiu S, Shao N and Zheng JH: LncRNA MALAT1 promotes proliferation and metastasis in epithelial ovarian cancer via the PI3K-AKT pathway. Eur Rev Med Pharmacol Sci 21: 3176-3184, 2017.

10. Gupta RA, Shah N, Wang KC, Kim J, Horlings HM, Wong DJ, Tsai MC, Hung T, Argani P, Rinn JL, et al: Long non-coding RNA HOTAIR reprograms chromatin state to promote cancer metastasis. Nature 464: 1071-1076, 2010.
11. Deng M, Bragelmann J, Schultze JL and Perner S: Web-TCGA: an online platform for integrated analysis of molecular cancer data sets. BMC Bioinformatics 17: 72, 2016.

12. R Core Team: R: A language and environment for statistical computing. R Foundation for Statistical Computing, Vienna, 2012. http://www.r-project.org/.

13. RStudio Team (2015). RStudio: Integrated Development for R. RStudio, Inc., Boston, MA, 2015. http://www.rstudio.com/.

14. Robinson MD, McCarthy DJ and Smyth GK: edgeR: A Bioconductor package for differential expression analysis of digital gene expression data. Bioinformatics 26: 139-140, 2010.

15. Tibshirani R: Regression shrinkage and selection via the lasso: A retrospective. J Royal Statist Soc Series B 73: 273-282, 2011.

16. Chan WL, Huang HD and Chang JG: IncRNAMap: A map of putative regulatory functions in the long non-coding transcriptome. Comput Biol Chem 50: 41-49, 2014.

17. Cao Z, Pan X, Yang Y, Huang Y and Shen HB: The lncLocator: A subcellular localization predictor for long non-coding RNAs based on a stacked ensemble classifier. Bioinformatics 34: 2185-2194, 2018.

18. Schmitt AM and Chang HY: Long noncoding RNAs in cancer pathways. Cancer Cell 29: 452-463, 2016.

19. Shen Y, Wang Z, Loo LW, Ni Y, Jia W, Fei P, Risch HA, Katsaros D and Yu H: LINC00472 expression is regulated by promoter methylation and associated with disease-free survival in patients with grade 2 breast cancer. Breast Cancer Res Treat 154: 473-482, 2015.

20. Wang KC and Chang HY: Molecular mechanisms of long noncoding RNAs. Mol Cell 43: 904-914, 2011.

21. Lee JT: Epigenetic regulation by long noncoding RNAs. Science 338: 1435-1439, 2012.

22. Yu Y, Yang J, Li Q, Xu B, Lian Y and Miao L: LINC00152: A pivotal oncogenic long non-coding RNA in human cancers. Cell Prolif 50: 2017.

23. Yue B, Qiu S, Zhao S, Liu C, Zhang D, Yu F, Peng Z and Yan D: LncRNA-ATB mediated E-cadherin repression promotes the progression of colon cancer and predicts poor prognosis. J Gastroenterol Hepatol 31: 595-603, 2016.

24. Ellinger J, Gevensleben H, Muller SC and Dietrich D: The emerging role of non-coding circulating RNA as a biomarker in renal cell carcinoma. Expert Rev Mol Diagn 16: 1059-1065, 2016.

25. Huang HW, Xie H, Ma X, Zhao F and Gao Y: Upregulation of LncRNA PANDAR predicts poor prognosis and promotes cell proliferation in cervical cancer. Eur Rev Med Pharmacol Sci 21: 4529-4535, 2017.

26. Bao X, Duan J, Yan Y, Ma X, Zhang Y, Wang H, Ni D, Wu S, Peng C, Fan Y, et al: Upregulation of long noncoding RNA PVT1 predicts unfavorable prognosis in patients with clear cell renal cell carcinoma. Cancer Biomark 21: 55-63, 2017.

27. Wang PQ, Wu YX, Zhong XD, Liu B and Qiao G: Prognostic significance of overexpressed long non-coding RNA TUG1 in patients with clear cell renal cell carcinoma. Eur Rev Med Pharmacol Sci 21: 82-86, 2017.

28. Xu Y, Tong Y, Zhu J, Lei Z, Wan L, Zhu X, Ye F and Xie L: An increase in long non-coding RNA PANDAR is associated with poor prognosis in clear cell renal cell carcinoma. BMC Cancer 17: 373, 2017.

29. Ning L, Li Z, Wei D, Chen H and Yang C: LncRNA, NEAT1 is a prognosis biomarker and regulates cancer progression via epithelial-mesenchymal transition in clear cell renal cell carcinoma. Cancer Biomark 19: 75-83, 2017.

30. Han L, Kong R, Yin DD, Zhang EB, Xu TP, De W and Shu YQ: Low expression of long noncoding RNA GAS6-AS1 predicts a poor prognosis in patients with NSCLC. Med Oncol 30: 694, 2013.

This work is licensed under a Creative Commons Attribution-NonCommercial-NoDerivatives 4.0 International (CC BY-NC-ND 4.0) License. 